\title{
An algebraic derivation of the distribution of rectangular coordinates
}

By P. L. Hsc.

Communicated by A. C. Aitkex.

(Received 20th April, 1940. Read 3rd May, 1940.)

Let $x_{i r}(i=1, \ldots, q ; r=1, \ldots, m ; q \leqq m)$ be random variables which have an elementary probability law $p\left(x_{11}, \ldots, x_{q m}\right)$. Let

$$
s_{i j}=\sum_{r=1}^{m} x_{i r} x_{j r}
$$

The fundamental assumption is that $p\left(x_{11}, \ldots, x_{q m}\right)$ is explicitly a function of the set of $s_{i j}$ alone, so that

$$
p\left(x_{11}, \ldots, x_{q m}\right)=f\left(s_{11}, s_{12}, \ldots, s_{q q}\right) .
$$

The $\frac{1}{2} q(q+1)$ functions $t_{i j}(i \leqq j)$, defined by the equation

where

$$
S=T T^{\prime \prime}
$$

$$
S=\begin{gathered}
s_{11} \ldots s_{1 q} \\
\ldots \ldots s_{q q} \\
s_{q 1} \ldots \ldots s_{q q}
\end{gathered}, T=\begin{gathered}
t_{11} 0 \ldots \ldots \\
t_{12} t_{22} \ldots \ldots 0 \\
\ldots \ldots \ldots \\
t_{1 q} t_{2 q} \ldots t_{q q}
\end{gathered}, t_{i i} \geqq 0
$$

and $T^{\prime}$ is the transposed matrix of $T$, are a generalisation of the rectangular coordinates of multivariate normal samples defined and studied by Mahalanobis and others in a joint paper ${ }^{1}$.

We have, directly from (2),

$$
s_{i j}=t_{1 i} t_{1 j}+t_{2 i} t_{2 j}+\ldots+t_{i i} t_{i j}
$$

To express the $t_{i j}$ in terms of the $s_{i j}$, we notice from (2) that, for $i \leqq j$,

$$
\begin{aligned}
& \begin{array}{ccc}
s_{11} \quad \ldots s_{1, i-1} & s_{1 j}
\end{array} \\
& \frac{\ldots \ldots \ldots \cdots \cdots \cdots}{s_{i-1,1} \ldots s_{i-1, i-1} s_{i-1, j}}= \\
& s_{i 1} \quad \ldots s_{i, i-1} \quad s_{i j}
\end{aligned}
$$

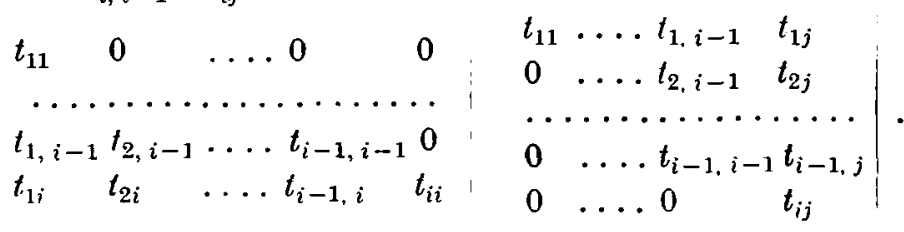

1 Mahalanobis, Bose and Roy, Srenlihya, 3 (1937), 1-40. This paper will be referred to as $(M)$. 
whence, taking determinants,

$$
\begin{aligned}
& s_{11} \quad \ldots s_{1, i-1} \quad s_{1 i} \\
& \begin{array}{c}
\ldots \ldots \cdots \cdots \cdots \cdots \\
s_{i-1,1} \ldots \ldots s_{i-1, i-1} s_{i-1, j}
\end{array}=t_{11}^{2} \ldots t_{i-1, i-1}^{2} t_{i i} t_{i j} . \\
& s_{i 1} \quad \ldots s_{i, i-1} \quad s_{i j}
\end{aligned}
$$

Setting $i=j$ in (4) we have

whence

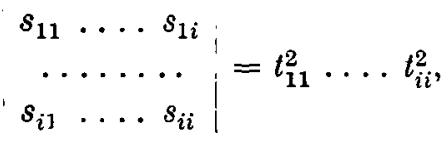

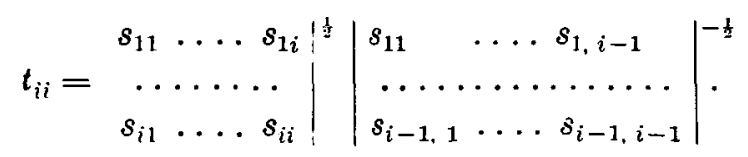

Dividing both sides of (4) by the corresponding sides of (5) and using (6) we obtain

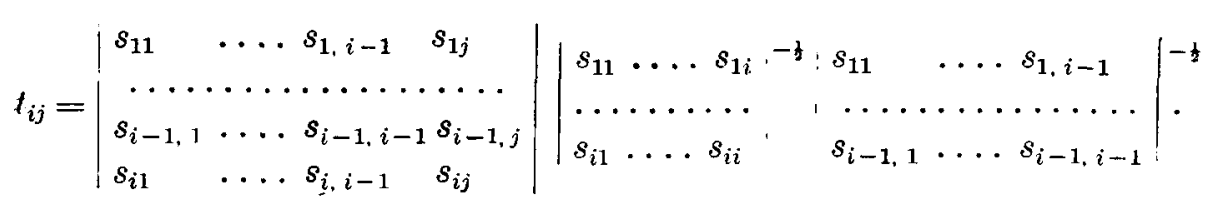

(3) and (7) are the relations connecting the rectangular coordinates $t_{i j}$ and the product moments $s_{i j}$ and have been derived in $(M)$.

In the case where the elementary probability law $p\left(x_{11}, \ldots, x_{q m}\right)$ is a normal one, a geometrical demonstration has been given in $(M)$ of the distribution of the $t_{i j}$. Here we shall give a purely algebraic derivation of the distribution, assuming that (1) holds true.

THEOREM. If (1) is true, the elementary probability law of the rectangular coordinates $t_{i j}$ defined by (2) is

$$
2^{q} \pi^{\frac{1}{2} m q-t q(q-1)}\left\{\prod_{i=1}^{q} \Gamma\left(\frac{1}{2}(m-i+1)\right)\right\}^{-1}\left(\prod_{i=1}^{q} t_{i i}^{m-i}\right) f\left(s_{11}, s_{12}, \ldots, s_{q q}\right),
$$

where the arguments of $f$ are to be regarded as the functions (3) of the $t_{i j}$.

Proof. By virtue of (1) we may express the elementary probability as

$$
f\left(s_{11}, s_{12}, \ldots, s_{q q}\right) d x_{11} \ldots d x_{q m} \text {. }
$$


Let us write more fully as follows:

$$
f\left[\begin{array}{c}
s_{11} \\
s_{12} s_{22} \\
\ldots \ldots \ldots \\
s_{1 q} s_{2 q} \ldots \ldots s_{q q}
\end{array}\right] d x_{11} \ldots . d x_{q m}
$$

Let the sets of variables $\left(x_{21}, \ldots, x_{2 m}\right), \ldots,\left(x_{q 1}, \ldots, x_{q m}\right)$ be subjected to the same linear transformation below whose coefficients are functions of $x_{11}, \ldots, x_{1 m}$ :

$$
\left.\begin{array}{l}
y_{i 1}=\left(\sum_{r=1}^{m} x_{1 r}^{2}\right)^{-t} \sum_{r=1}^{m} x_{1 r} x_{i r} \\
y_{i 2}=\sum_{r=1}^{m} c_{2 r} x_{i r} \\
\ldots \ldots \ldots \ldots \ldots, \ldots \ldots, \ldots \\
y_{i m 2}=\sum_{r=1}^{m} c_{m r} x_{i r}
\end{array}\right\},(i=2, \ldots,
$$

where the c's are so determined as to make (10) an orthogonal transformation. As the Jacobian is 1 , we get the result

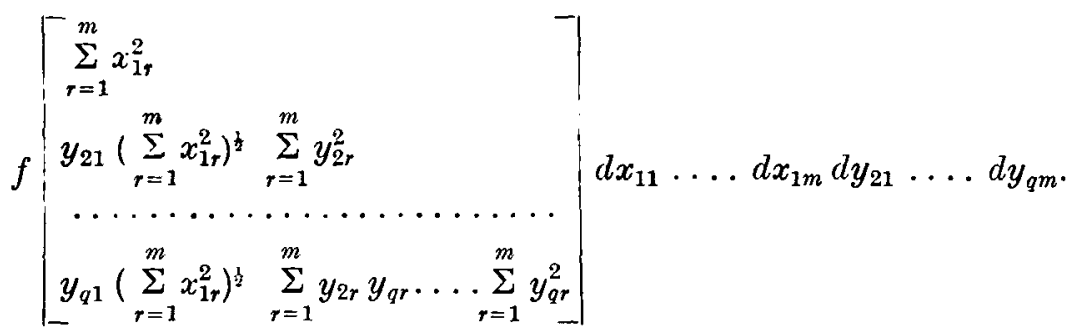

If for $x_{11}, \ldots, x_{1 m}$ we substitute spherical coordinates, viz. the radius vector, $t_{11}$, and $m-1$ angles, $\theta_{12}, \ldots, \theta_{1 m}$, we obtain

$$
\begin{aligned}
& t_{12}^{m-1}\left\{\prod_{a=3}^{m}\left(\cos \theta_{1 a}\right)^{a-2}\right\} f\left[\begin{array}{l}
l_{11}^{2} \\
t_{11} y_{21} \sum_{r=1}^{m} y_{2 r}^{2} \\
\ldots \ldots \ldots \ldots \ldots \ldots \ldots
\end{array}\right] d t_{11} d \theta_{12} \ldots d \theta_{1 m} d y_{21} \ldots d y_{q m} . \\
& t_{11} y_{q 1} \sum_{r=1}^{m} y_{2 r} y_{q r} \ldots \sum_{r=1}^{m} y_{q r}^{2}
\end{aligned}
$$

Writing $t_{1 i}$ for $y_{i 1}(i=2, \ldots q)$ and

$$
{s^{\prime}}_{i j}=\sum_{r=2}^{m} y_{i r} y_{j r}(i, j=2, \ldots, q) \text {, }
$$


we get

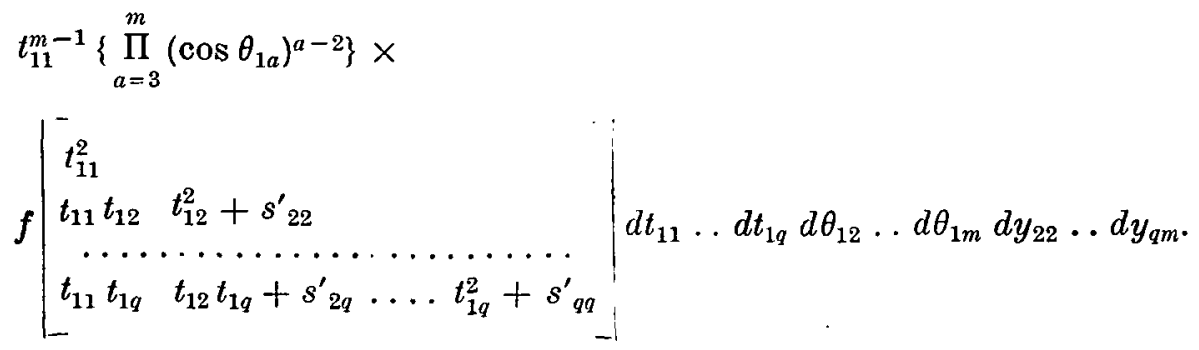

It is seen that, as far as the $y$-variables are concerned, we have the same situation that only the product moments $s^{\prime}{ }_{i j}$ figure in the elementary probability law. Hence the same procedure which carries (9) to (11) may be repeated. In doing so we introduce the following variables: $t_{22}, t_{23}, \ldots, t_{2 q} ; \theta_{23}, \ldots, \theta_{2 q} ; z_{33}, z_{34}, \ldots, z_{q m}$, to replace the $y$ 's, and write down the elementary probability:

$$
\begin{aligned}
& t_{11}^{m-1} t_{22}^{m-2}\left\{\prod_{a=3}^{m}\left(\cos \theta_{1 a}\right)^{a-2}\right\}\left\{\prod_{a=4}^{m}\left(\cos \theta_{2 a}\right)^{a-3}\right\} \times
\end{aligned}
$$

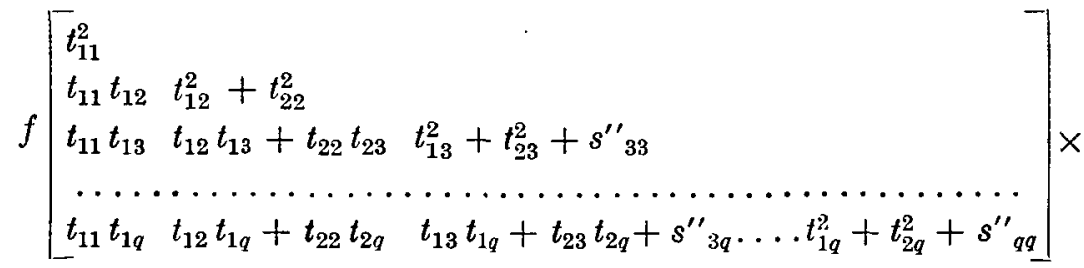

$d t_{11} \ldots d t_{1 q} d t_{22} \ldots d t_{2 q} d \theta_{12} \ldots d \theta_{1 m} d \theta_{23} \ldots d \theta_{2 m} d z_{33} \ldots d z_{q m}$,

where

$$
s^{\prime \prime}{ }_{i j}=\sum_{r=3}^{m} z_{i r} z_{j r}(i, j=3, \ldots, q) .
$$

Proceeding in this manner we finally obtain

$$
\begin{aligned}
& \left(\prod_{i=1} t_{i i}^{m-i}\right)\left\{\prod_{i=1}^{q} \prod_{a=i+2}^{m}\left(\cos \theta_{i a}\right)^{a-i-1}\right\} \times \\
& \bar{t}_{11}^{2} \\
& f^{t_{11} t_{12}} t_{12}^{2}+t_{22}^{2} \\
& t_{11} t_{1 q} t_{12} t_{1 q}+t_{22} t_{2 q} \ldots t_{1 q}^{2}+t_{2 q}^{2}+\ldots+t_{q q}^{2} \\
& d \theta_{12} \ldots d \theta_{q+1, m} d t_{11} d t_{12} \ldots d t_{q q} .
\end{aligned}
$$

Now the functions $t_{i j}$ introduced in the proof are precisely the rectangular co-ordinates defined by (2) or (3). For, in each step of transformation from (9) to (12) the change in the arguments of $f$ is 
effected by direct substitution. Equating the arguments of $f$ in (9) and (12) we get

$$
s_{i j}=t_{1 i} t_{1 j}+t_{2 i} t_{2 j}+\ldots+t_{i i} t_{i j}
$$

which gives (3).

If from (12) the $\theta$ 's are integrated over the following domain:

$$
\begin{array}{ll}
-\pi \leqq \theta_{i a} \leqq \pi & (a=i+1 ; i=1, \ldots, q), \\
-\frac{1}{2} \pi \leqq \theta_{i a} \leqq \frac{1}{2} \pi & (a=i+2, \ldots, m ; i=1, \ldots, q),
\end{array}
$$

the result is the elementary probability law (8). The proof is therefore complete.

One more step leads to the distribution of the product moments $s_{i j}$, as is done in $(M)$. The transformation is given by (7) and the reciprocal transformation by $(3)$, which has the Jacobian

$$
2^{q} t_{11}^{q} t_{22}^{q-1} \ldots t_{q q}
$$

Dividing (8) by this Jacobian and then carrying out the substitution we get the following elementary probability law of the $s_{i j}$ :

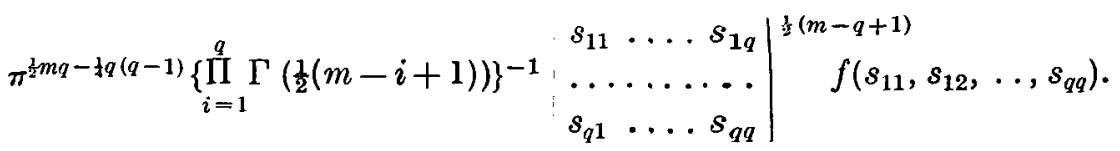

The multiplier of $f\left(s_{11}, s_{12}, \ldots, s_{q q}\right)$ in $(13)$ is well known ${ }^{1}$.

The result (12) brings out the following important fact: If $x_{11}, x_{12}, \ldots, x_{q m}$ are all the observational data and if only the rectangular coordinates $t_{i j}$ or the product moments $s_{i j}$ are utilised for statistical purposes, the part of the observational data thus thrown away may be regarded as a set of angles which are distributed independently of the $t_{i j}$ or $s_{i j}$ and whose elementary probability law does not involve any of the unknown parameters that may figure in the elementary probability law of the $x$ 's.

${ }^{1}$ Cf. Wishart and Bartlett, Proc. Camb. Phil. Soc., 29 (1933), 271-6.

153, Chesterton Road, Camiridge. 